\title{
Best of Echo für die Lungenhochdruck-Prognose
}

Fragestellung: Die Echokardiografie wird bei Verdacht auf pulmonale Hypertonie (PH) und der Verlaufskontrolle eingesetzt. Aber welche Parameter sind für die Verlaufsbeurteilung optimal?

Hintergrund: Die Identifikation von Patienten mit dem Risiko für eine Verschlechterung ihrer PH ist essenziell. Die Analyse soll die am besten untersuchten echokardiografischen Befunde identifizieren, die mit der PAH-Verschlechterung assoziiert sind.

Methode: Die MEDLINE- und EMBASE-Datenbanken wurden nach Longitudinalstudien durchsucht, die die Assoziation von echokardiografischen Befunden zu Mortalität, Transplantation und klinischer Verschlechterung prüften und bis April 2015 veröffentlicht wurden. Die Metaanalyse basierte auf dem „random effect model“, um die Aussage von echokardiografischen Ergebnissen in mindestens 4 Studien zu prüfen. Im Fall von statistischer Heterogenität erfolgten Sensitivitätsanalysen.

\section{Originalie}

Baggen VJ, Driessen MM, Post MC et al. Echocardiographic findings associated with mortality or transplant in patients with pulmonary arterial hypertension. Neth Heart J. 2016;24(6):374-89.

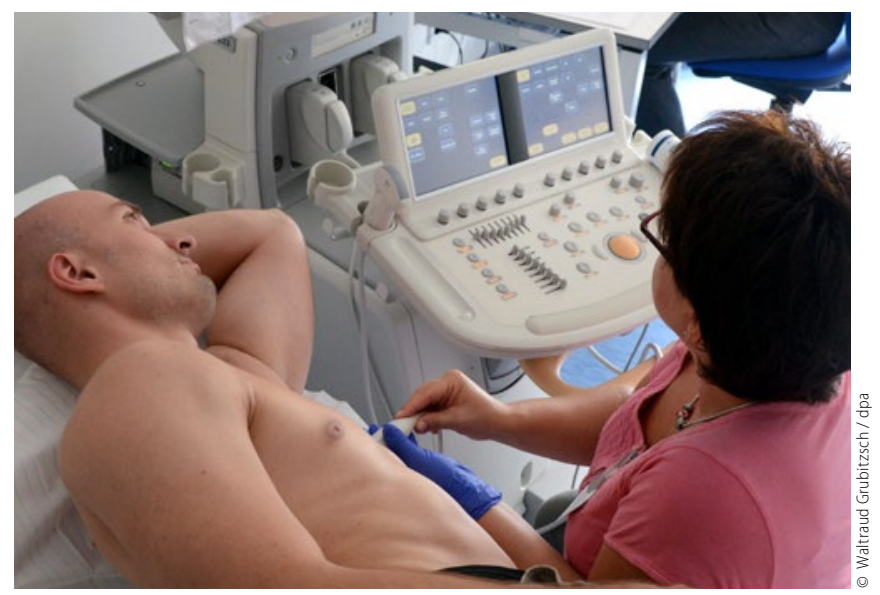

Vorhof, Trikuspidalklappe und Perikard geben beim Herzecho die zuverlässigste Auskunft, wie es bei pulmonaler Hypertonie steht.

die Zunahme der rechten Vorhoffläche (pooled HR 1,71; 95\%KI 1,38-2,13) und die Verminderung der tricuspid annular plane systolic excursion (TAPSE; pooled HR 1,72; 95\%-KI 1,34$2,20)$ die meist untersuchten und robustesten Prädiktoren für eine Verschlechterung und für eine Transplantation waren.

Schlussfolgerungen: Diese Metaanalyse spricht für den Nutzen von echokardiografischen Verlaufsuntersuchungen bei PH-Patienten, v.a. die Wichtigkeit der oben genannten 3 Befunde.

\section{- Kommentar von Dr. med. Stephan Holt}

\section{Drei robuste Prädiktoren mit geringer Observer-Variabilität}

In der Metaanalyse wurde das ganze Spektrum der pulmonalen Hypertonie von der PH bei Lungenerkrankungen (Nizza Klassifikation 3), über die chronisch thromboembolische $\mathrm{PH}$ (CTEPH, Nizza Klassifikation 4) bis zur PH bei kongenitalen Herzfehlern untersucht. Sie berücksichtigte aber v.a. Patienten mit $\mathrm{PAH}$. Die Patienten waren 31-61 Jahre alt (60\%-98\% Frauen) und $35-100 \%$ in fortgeschrittenen Stadiem der Erkrankung (NYHA III-IV). Die Patientenzahlen variierten mit 25-2716 je Studie stark. In allen Studien wurde die PH mittels Rechtsherzkatheter diagnostiziert, nur in einer wurde sie bei $87 \%$ der Teilnehmer mittels Rechtsherzkatheter und 13\% nur mittels Echokardiografie diagnostiziert. Somit ist die PH exakt diagnostiziert worden und es wird ein breites Spektrum betrachtet.

Das Neuauftreten eines Perikardergusses, eine Abnahme der TAPSE um mindestens $5 \mathrm{~mm}$ und eine Zunahme der rechtsatrialen Fläche um mindestens $5 \mathrm{~cm}^{2}$ ist mit einer signifikant höheren Mortalität assoziiert. Viele weitere echokardiografischer Parameter wurden geprüft, aber die genannten 3 sind die in allen Studien am Besten untersuchten und zeigten die größte prognostische Bedeutung. Dies entspricht den Resul- taten der bisherigen Studien. Die Bedeutung einer Zunahme der Fläche des rechten Vorhofes und der Abnahme der TAPSE um mindestens $5 \mathrm{~mm}$ wird hier aber noch einmal besonders herausgestellt.

Alle 3 Parameter sind schnell und einfach zu untersuchen und haben so in unserer Ambulanz für $\mathrm{PH}$ eine große Bedeutung als Verlaufsparameter. Alle 3 Werte haben eine geringe Intra- wie Interobserver-Variabilität und sollten so von jedem Untersucher reproduzierbar ermittelt werden können.

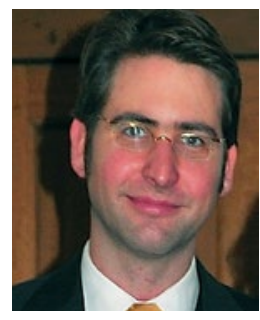

Dr. med. Stephan Holt

Krankenhaus Bethanien

Klinik für Pneumologie und Allergologie Aufderhöher Str. 169-175, 42699 Solingen Stephan.Holt@Klinik-Bethanien.de 